\title{
Size Distribution of Inorganic Species and Their Inhaled Dose in a Detergent Industrial Workplace
}

\author{
A. Karanasiou $\cdot$ K. Eleftheriadis $\cdot$ S. Vratolis • \\ P. Zarbas • N. Mihalopoulos • C. Mitsakou • \\ C. Housiadas • M. Lazaridis • J. Ondracek • \\ L. Dzumbova
}

Published online: 6 December 2007

(C) Springer Science + Business Media B.V. 2007

The following Acknowledgement should have been mentioned in the paper entitled Size Distribution of Inorganic Species and Their Inhaled Dose in a Detergent Industrial Workplace by A. Karanasiou, K. Eleftheriadis, S. Vratolis, P. Zarbas, N. Mihalopoulos, C. Mitsakou, C. Housiadas, M. Lazaridis, J. Ondracek and L. Dzumbova:

Acknowledgment The work is partially supported by GSRT through grant from the O.P. "Competitiveness" (Measure 4.5, Action 4.5.1). This project is co-funded (75\%) by the European Union - European Regional Development Fund (ERDF).

The online version of the original article can be found at http:// dx.doi.org/10.1007/s11267-007-9140-z.

\footnotetext{
A. Karanasiou $\cdot$ K. Eleftheriadis $(\bowtie) \cdot$ S. Vratolis $\cdot$ C. Mitsakou $\cdot$ C. Housiadas Institute of Nuclear Technology \& Radiation Protection, N.C.S.R. "Demokritos", 15310 Ag. Paraskevi, Attica, Greece

e-mail: elefther@ipta.demokritos.gr

M. Lazaridis · J. Ondracek · L. Dzumbova Department of Environmental Engineering, Technical University of Crete, 73100 Chania, Crete, Greece

P. Zarbas $\cdot$ N. Mihalopoulos

Environmental Chemical Processes Laboratory, Department of Chemistry, University of Crete, P.O. Box 1470, 71409 Heraklion, Greece
} 